\title{
Deep-Analysis of Palmprint Representation Based on Correlation Concept for Human Biometrics Identification
}

\author{
Raouia Mokni, College of Computer Engineering and Science, Sattam Prince Bin Abdulaziz University, Kharj, Saudi \\ Arabia \& Faculty of Economics and Management of Sfax, University of Sfax, Sfax, Tunisia \\ Hassen Drira, IMT Lille Douai, Univ. Lille, CNRS, UMR 9189 -CRIStAL, Lille, France \\ Monji Kherallah, Faculty of Sciences of Sfax, University of Sfax, Sfax, Tunisia
}

\begin{abstract}
The security of people requires a beefy guarantee in our society, particularly, with the spread of terrorism throughout the world. In this context, palmprint identification based on texture analysis is amongst the pattern recognition applications to recognize people. In this article, the researchers investigated a deep texture analysis for the palmprint texture pattern representation based on a fusion between several texture information extractions through multiple descriptors, such as HOG and Gabor Filters, Fractal dimensions and GLCM corresponding respectively to the frequency, model, and statistical methodologies-based texture features. They assessed the proposed deep texture analysis method as well as the applicability of the dimensionality reduction techniques and the correlation concept between the features-based fusion on the challenging PolyU, CASIA and IIT-Delhi Palmprint databases. The experimental results show that the fusion of different texture types using the correlation concept for palmprint modality identification leads to promising results.
\end{abstract}

\section{KEYWORDS}

Biometric, Classification, Correlation Concept, Deep-Analysis, Fusion, GDA, MCCA, Palmprint, Random Forest

\section{INTRODUCTION}

The need for automated secure access to physical or virtual environments, especially for personalized services, is growing. These needs require reliable means to verify the identity of a person who is having access to the system. However, conventional means, such as passwords or magnetic cards associated with a personal code, have a number of shortcomings. For instance, a password may be forgotten or stolen by another individual, or even given to someone else and access cards may also be lost or stolen.

This is how the science of automatic identification of people through the exploitation of their unique physical and behavioral traits, such as face, signature, voice, fingerprints, shape of the hand, palmprint, etc., appeared the most reliable solution. Each of these different traits is called biometric modality.

Among these physical modalities, we are interested in the palmprint, which is the most mature biometric technology that has been used for more than a century. Hitherto, more attention has been paid to palmprint identification systems through research teams because it presents diverse advantages 
compared to other modalities in the biometric system such as its usability, stability over time, low cost, affordable materials, high recognition accuracy, etc.

The palmprint is particularly solicited in biometrics: it is the inside of the hand between the wrist and the roots of the fingers. It illustrates the most discriminating physical characteristic for the recognition of its skin patterns including both the principal lines and the texture representations, which are appropriated for the use of the real application within palmprint recognition.

In fact, an overview of the related work methods about palmprint identification applications is presented. Till now, these already existing applications can be divided into two popular categories, Global approach and Structural approach, based mainly on the extraction and the analysis of the different palmprint representations.

Structural approaches: They analyze the structure of the palmprint and include the palmprint lines, such as the principal lines ( $c f$. ., (Han, Cheng, Lin, \& Fan, 2003), (Mokni, Drira, \& Kherallah, 2017a), etc.), the wrinkles ( $c f$. ., (Chen, Zhang, \& Rong, 2001), etc.), the ridges and minutiae ( $c f$. , (Duta, Jain, \& Mardia, 2002), etc.) for represented the palmprint pattern.

Although this type of approach relies on taking into account the structural particularity of the palmprint representations since it is stable over time, unluckily, those representations alone cannot provide satisfactory information to identify the person efficiently.

Global approaches: They use the whole area of the palmprint as input to their recognition algorithm. Over recent years, researchers have been interested in this type of approach that presents different descriptors or methods to analyze the texture pattern of an image. In fact, different proposals have been presented. We distinguish three methodologies based on the texture feature analysis such as Statistical, Frequency and Model. Each one of them involves various descriptors.

In fact, (1) Statistical methodology includes Eigenpalms (cf.,(Lu, Zhang, \& Wang, 2003), etc.), Fisherpalms ( $c f$., (Wu, Zhang, \& Wang, 2003), etc.), Local Binary Pattern (LBP) ( $c f$. ., (Hammami, Ben-Jemaa, \& Ben-Abdallah, 2014), etc.), Gray Level Co-occurrence Matrix (GLCM) (cf., (Latha \& Prasad, 2015)), (Mokni, Elleuch, \& Kherallah, 2016), etc.), (2) Frequency methodology involves different descriptors such as Gabor filters (GF) ( $c f$., (Kumar \& Shekhar, 2010), (Ben-Khalifa, Rzouga, \& BenAmara, 2013), etc.), Scale-Invariant Feature Transform (SIFT) (cf., (Hammami et al., 2014)), Wavelets ( $c f$. ., (Kekre, Sarode, \& Tirodkar, 2012), etc.), and (3) Model methodology groups Blanket or Fractal, Multi-Fractal dimensions ( $c f$. ., (Guo, Zhou, \& Wang, 2014), etc.).

Some research studies mentioned above proposed several approaches that used the Statistical and the Frequency methodologies-based texture feature, separately ( $c f$. ., (Hammami et al., 2014) and (Ben-Khalifa et al., 2013).

Although some of the palmprint biometric systems based on a single Analysis of its features have reached a performance which seems efficient for recognition, they have some issues that make them useless in all current applications. These issues can emerge in numerous problems due to uncontrolled environmental conditions, lack of representation individuality (e.g., intra-class variation, inter-class similarities), lack of security level (e.g., fraud attacks or spoofing), and so on.

There are several challenging problems related to the texture pattern, which include, for instance, the intra-class variations and inter-class similarity of the texture skin pattern affected by the aging, contraction, stretching and lighting, etc. Any variability can be a major source of difficulty of person's identification in a real application. To deal with some of these problems and increase the level of security, a deep analysis, which is based on the combination of the information presented by several analyses of texture representation, may allow a more precise identification of the identity. This is called Intra-Modal biometric system based on Multi-Analyses of texture features for palmprint biometric system. 
Thus, in this paper we propose to fuse several texture features extracted through multiple descriptors belonging to different analysis methodologies for efficient palmprint identification. The main contributions of this work are the following:

- Global feature representing the whole texture part of the palmprint having more pertinent features as an input to our palmprint biometric recognition algorithm. Actually, the previous texture information analysis-based approaches did not investigate the deep analysis of its features incorporating the correlation concept between them. For this reason, we describe the palmprint image by multiple descriptors that better characterize the texture pattern information;

- These descriptors are invariant to different variations produced in an unconstrained environment. They ensure a simple calculation enabling to analyze the images in real time applications. Therefore, the texture information is investigated using three methodologies based on the texture features analysis, like Model, Statistical and Frequency. In so doing, the palmprint texture is firstly extracted based on the Frequency methodology relying on the HOG and GF descriptors. Moreover, it is also analyzed based on the Model and Statistical methodologies relying on Fractal Dimension and GLCM descriptors, respectively;

- Four feature vectors which correspond to the three proposed methodologies are extracted. Some of these vectors have high dimensions, which leads to the dimensionality problems. In order to deal with this problem, we applied dimensionality reduction techniques named such as Principal Component Analysis (PCA) or Generalized Discriminant Analysis (GDA) in order to increase the performance and decrease the computational complexity;

- Fusion between the texture information analysis methodologies, like Model, Statistical and Frequency, is applied. The used descriptors belonging to different methodologies of texture analysis give multiple independent features which have different types of information;

- This fusion between these features as well as the incorporation of the correlation analysis between them using the fusion at feature level, namely Multi-set Correlation Canonical Analysis method, produce a complete, relevant and discriminant texture analysis;

- As far as classification is regarded, we advocate employing the multi-class version of both the SVM and Random Forest classifiers;

- An extensive empirical analysis involving three famous datasets which are PolyU-Palmprint, CASIA-Palmprint and IIT-Delhi- Palmprint datasets.

The reminder of this paper is structured as follows: In Sect.2, the overview of the proposed deep analysis approach and its phases are detailed. The used datasets and the experimental results in terms of accuracy of palmprint identification in comparison with state-of-the-art methods are described in Sect.3. Lastly, a conclusion and some future research directions are discussed in Sect.4.

\section{OUR PROPOSED APPROACH}

An overview of the proposed approach is given in Figure 1 that displays several followed phases in our palmprint identification system such as (1) Hand Palmprint Acquisition (2) Palmprint Preprocessing (3) Feature Extraction (4) Feature vectors dimensionality reduction, (5) Feature Fusion, (6) Classification and (7) Decision.

Therefore, we first use the palmprint image from dataset. Then, we apply the preprocessing process on the palmprint image in order to extract the region of interest having the most discriminatory features for identification. Next, several analyses are applied using several texture analysis methodologies (e.g., Frequency, Model and Statistical methodologies) based on multiple descriptors (e.g., HOG, Gabor, Fractal Dimension and GLCM descriptors). After that, several dimensionality reduction techniques (PCA and GDA) are used in order to pick up the pertinent features, reduce the length of the feature vectors and their redundancies. Successively, fusion at feature level based on Multi-set 
Canonical Correlation Analysis (MCCA) technique is performed to build the final feature vector. Finally, the palmprint identification is performed by Support Vector Machine (SVM) and Random Forest (RF) classifiers.

In the following subsections, we will detail each of these phases while describing its different basic used concepts and techniques.

\section{Palmprint Preprocessing}

The palmprint preprocessing is a paramount phase in the general biometric system. It is devoted for extracting the Region of interest (ROI) which has discriminatory and important features of recognition. This module includes three main steps (see Figure 2), namely: Edge detection of the hand, Coordinate system Stabilization and ROI Preprocessing, which are described in detail in (Mokni \& Kherallah, 2016a), (Mokni, Mezghani, Drira, \& Kherallah, 2017).

In the following, we briefly remind these steps:

1. Edge detection of the hand: The first step of the preprocessing module aims to extract the hand edge in order that we can localize the finger-webs addressing to stabilize the ROI. So, we firstly converted the input hand image into binary image using an automatic thresholding technique such as the Otsu technique (Otsu, 1979). Then, we employed the Morphology operations incorporating the closing and the opening operations in order to avoid some identification problems which are founded when the users place their hands in front of the camera with wearing accessories as the rings. Finally, we detected the contour of the hand and applied the smoothing filter;

2. Coordinate System Stabilization: The second step focuses on the stabilization of the coordinate system in order to extract the ROI. For this end, we begin by adjusting the hand image with its contour and detecting its centroid as a reference point. Subsequently, we extracted the fingerwebs in order to obtain a stable coordinate system which enables us to extract and locate the ROI square;

Figure 1. Overview of our proposed approach

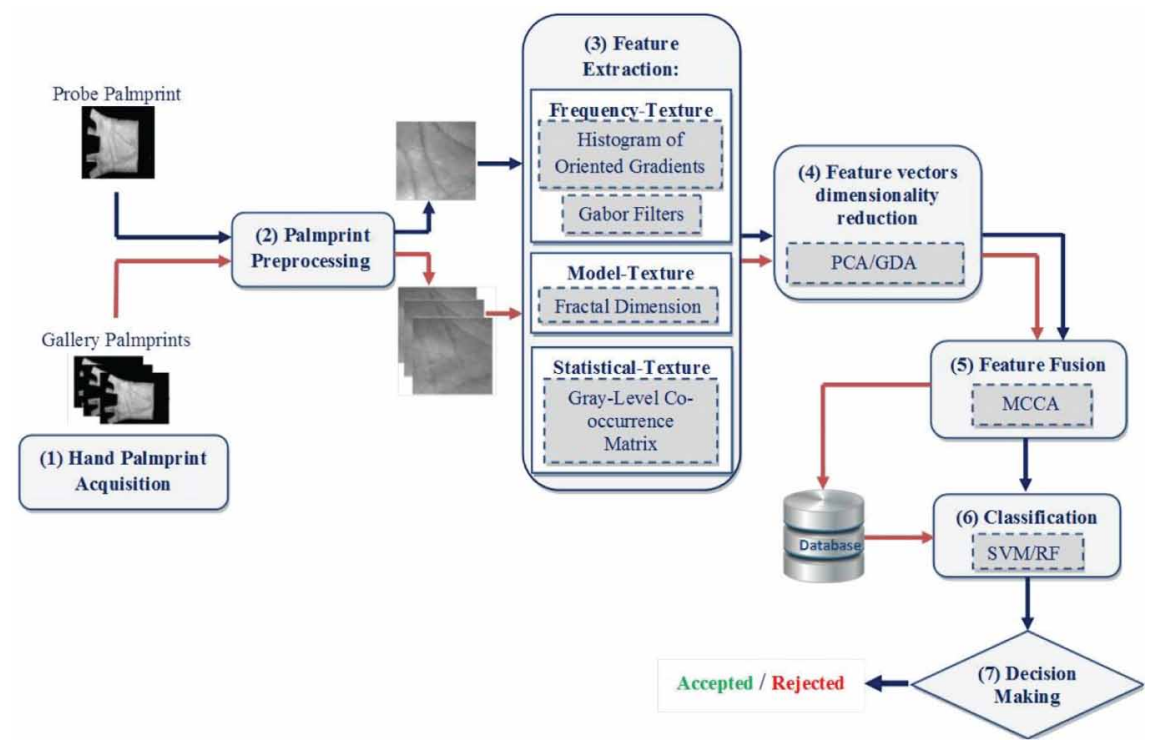


3. ROI Preprocessing: After the ROI square extraction and localization, it is necessary to fix its size to $S \times S$ with $S=128$ pixels and then rotate it to a vertical position. Lastly, we applied a low pass filter to enhance the quality of ROI in order to restrict the noise.

\section{Deep Analysis of Palmprint Texture Representation}

Deep analysis based on the Multi analyses of the texture representation features of palmprint modality is conducted using different descriptors. Then, a fusion between the extracted features is performed; so that we can achieve an effective recognition solution to identify the persons. These descriptors show their robustness against the numerous challenges and variation of palmprint produced in unconstrained environments.

\section{Feature Extraction Based on Multiple Analyses}

In this section, we briefly described the different feature extractors used in the present work for palmprint texture analysis studies such as the Histograms of Oriented Gradients (HOG) (Dalal \& Triggs, 2005), Gabor Filter (GF) (Lee, 1996), the Fractal Dimension (FD) (Mandelbrot, 1975) and the Gray Level Concurrence Matrix (GLCM) (Haralick, 1979) descriptors.

These descriptors characterize and analyze the texture pattern and show their robustness against the different transformations in terms of position, lighting variations and texture deformation of palmprint that are produced in an unconstrained environment during the acquisition of an image.

\section{Frequency Methodology Based on Histograms of Oriented Gradients Descriptor (HOG)}

HOG descriptor is introduced by Dalal and Triggs (Dalal \& Triggs, 2005) to extract the local appearance and shape of objects (human body). This descriptor is also considered as one of the efficient frequency methods used to extract the relevant texture features of the image in literature. The main objective of these extracted features is their invariance to different object transformations and variations (e.g., texture deformation, lighting conditions, etc.).

In our proposed system, in order to detail the process of calculating the Histogram of Oriented Gradient, we are initially described as the distribution or histograms of the orientation of gradients which means to the distribution of the local intensity gradients or edge directions of an image being calculated from small connected regions.

Figure 2. The Palmprint Preprocessing: (1) Edge detection of the hand; (2) Coordinate system stabilization; and (3) ROI preprocessing
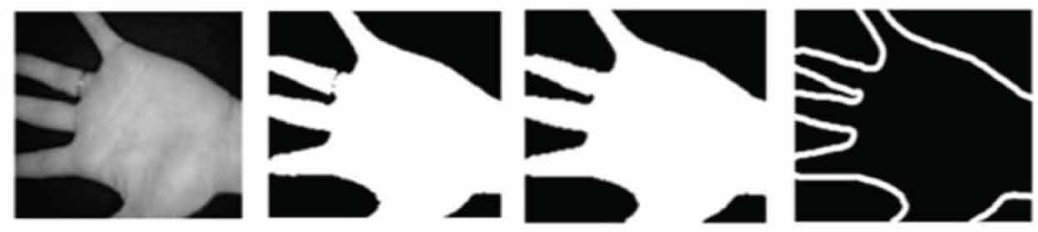

(1) Edge Detection of the Hand
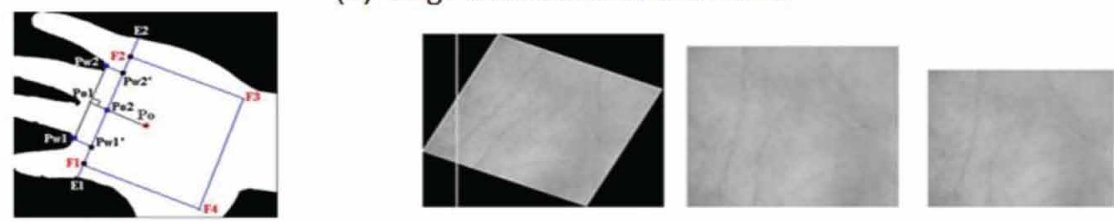

(2) Coordinate System Stabilization

(3) ROI Preprocessing 
To this end, practically, the image window is divided into small connected spatial regions named cells, for each cell assembling a local 1-D histogram of gradient directions or edge orientations (1-D gradient filter mask [ $\left.\left[\begin{array}{lll}-1 & 0 & 1\end{array}\right]\right)$ over the pixels of the cell. The combined histogram entries present the representation. The gradients of an image are sensitive to overall lighting. In fact, for better invariance to illumination, shadowing, the local histograms were contrast-normalized by calculating a measure of the local histogram "energy" over a larger region of the image, called blocks, and hence utilizing this value to normalize all cells inside the block. This process aims to normalize the histogram, so it is not affected by lighting variations. Note that these blocks typically overlap. This normalized descriptor can be referred as Histogram of Oriented Gradient (HOG) descriptors. Four types of normalization are explored: L2-norm, L2-Hys, L1-sqrt, and L1-norm. In our experiment the L2-norm scheme was employed thanks to its more used factor in pattern recognition application:

$$
L 2-\text { norm }: \quad F=\frac{v}{\sqrt{\|v\|_{k}^{2}}+\varepsilon^{2}}
$$

where $\mathrm{v}$ is he non-standard vector containing all the self-contained histograms, his k-norm is designed by $\sqrt{\|v\|_{k}}, \varepsilon$ is a constant of low value and $\mathrm{F}$ is the normalized HOG descriptor feature vector.

There exist two main blocks: Rectangular R-HOG blocks and Circular C-HOG blocks. In our experiments, the HOG descriptor is calculated over R-HOG blocks that are the square grids.

During the calculation of the HOG vector features, it can be noted that each pixel within the image aids in the magnitude of its gradient to a histogram orientation bin which compatible to the orientation of its gradient. The gradient orientations are accumulated into 9 orientation bins. The descriptor of the object of interest would then be represented by the histograms. The feature descriptor is measured by the combination of the histograms from each block. Therefore, the feature vector dimension of the HOG descriptor is computed although the multiplication between the selected numbers of the blocks and the orientation bins. Practically, we have divided the image into $10 \times 10$ rectangular blocks and 9 bin histograms per blocks. The $10 \times 10$ blocks with nine bins were then concatenated to make a 900-dimensional feature vector: $($ FVHOG $=(10 \times 10) \times 9=900)$.

\section{Frequency Methodology Based on Gabor Filter Descriptor}

The Gabor Filter (GF) is one of the earliest and valuable frequency methods used to analyze the texture of the image. The mainly advantage of GF focuses on their different challenging and variability concerning the rotation, position, translation, scale and lighting conditions of palmprint texture image in unconstrained environments. The Gabor filter is based on extracting directly the texture features from the Gray level images. In the spatial domain, a 2-D Gabor filter based in both frequency (scale) and orientation is a Gaussian kernel function modulated by a complex sinusoidal plane wave, defined as the following equation:

$$
\begin{aligned}
& G_{F}(x, y)=\frac{\mu^{2}}{\pi \times \gamma \times \eta} \exp \left(-\left(\frac{x^{\prime 2}+\gamma^{2} \times y^{\prime 2}}{2 \sigma^{2}}\right)\right) \exp \left(j \times 2 \times \pi \times f \times x^{\prime}+\phi\right) \\
& x^{\prime}=x \cos \nu+y \sin \nu \\
& y^{\prime}=-(x \sin \nu+y \cos \nu)
\end{aligned}
$$

where $\mu$ represents the central frequencies (scale) of the sinusoidal plane wave and $\nu$ is the orientation of the normal to the parallel stripes of a Gabor function. $\sigma$ is the standard deviation of the Gaussian 
envelope, $\phi$ is the phase offset and $\gamma$ is the ratio of the spatial aspect that stipulates the ellipticity of the Gabor function support. In our experimental evaluations, the palmprint image is convolved with $\mathrm{G}=40$ Gabor filters at five frequencies $(\mu=5)$ and eight orientations $(\nu=8)$, as illustrated in Figure 3. We pursue to apply the same Gabor filters parameters suggested by Haghighat et al. (Haghighat, Zonouz, \& Abdel-Mottaleb, 2015) to build the feature vector. Thus, let us define that we calculated the vector dimension as:

$$
F V_{G F}=((S \times S) \times \mu \times \nu) /(d \times d)
$$

where $S=128$ pixels is the size of palmprint ROI image, $\mu=5$ is the number of frequencies, $\nu=8$ is the number of orientations and $d=4$ is the factor of downsampling the features that is used to reduce the redundancy of the texture information producing by the correlation between the adjacent pixels in palmprint ROI image. Therefore, the dimension of the feature vector is equal to $(128 \times 128 \times 40) /$ $(4 \times 4)=655,360 / 16=40960$. In addition, this vector is normalized to zero mean and unit variance.

\section{Model Methodology Based on Fractal Dimension Descriptor}

Fractal Dimension (FD) method is one of the effective methods used to extract the Model based texture features. It is considered as a widely applied descriptor, especially for analyzing the texture representation, in several fields like the signature recognition (Zouari, Mokni, \& Kherallah, 2014), palmprint recognition (Mokni \& Kherallah, 2016b), written identification (Chaabouni, 2014), etc.

A fractal object has a very complicate shape which is a collection of different sub-objects recurring in different scales. Several methods are proposed to compute the fractal dimension like the Box Counting (BC), Differential Box Counting (DBC), Mass Radius (MR), Cumulative Intersection (CI), etc. Among these methods, the BC is the deeply used method to estimate the fractal dimension and analyze the texture shape thanks to its utility to describe the interruption and complexity of the image texture.

The ultimate principle of $\mathrm{BC}$ method focuses on converting the input image to binary image and then splitting it into a boxes number with several scales $\varepsilon$. After that, it is necessary to compute the number of boxes including the shape information $N(\varepsilon)$ for each scale. Thus, the fractal dimension $F D$ is obtained by a least squares regression method, as illustrated in the following equation:

Figure 3. Applying Gabor filter descriptor on palmprint image: (a) The extracted palmprint ROI; (b) The real part with applying the Gabor filter in five frequencies and eight orientations on the palmprint image

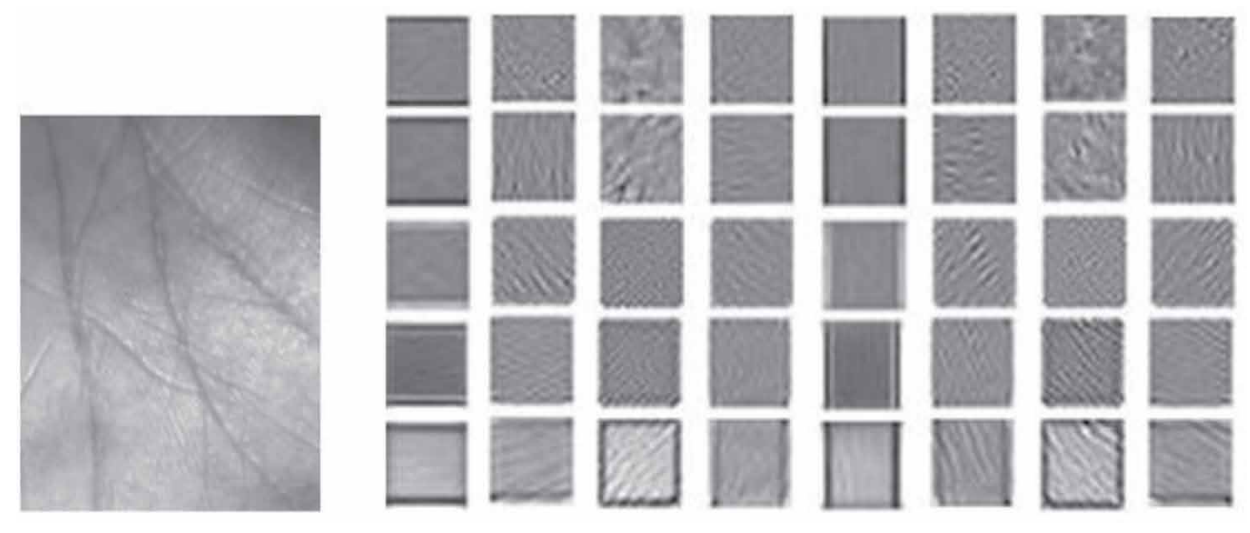




$$
F D=\text { slope }\left(\frac{\log (N(\varepsilon))}{\log \left(\frac{1}{\varepsilon}\right)}\right)
$$

In our experiments, we proposed a method to provide the shape information in horizontal and vertical spaces of palmprint ROI image. In this case, we divided it, sequentially, into $r \times r$ sub regions equivalent squares. It is imminent that the value $r=10$ is chosen after a set of experiments. For each sub-region, the fractal Dimension $F D$ is calculated.

Therefore, we obtained a sequence of $F D s$ for the whole input palmprint ROI image presented by the following feature vector:

$$
F V_{F D}=\left(F D_{1}, F D_{2}, \ldots, F D_{r \times r}\right)
$$

\section{Statistical Methodology Based on Gray Level Concurrence Matrix Descriptor}

The Co-occurrence Matrix (GLCM) is one of the robust statistical methods used to describe the image texture. This method presents a matrix which contains valuable information about the statistical distribution of intensities and provides information about the relative position of neighborhood pixels of the analyzed image.

More specifically, let us have an image Img, of size $S \times S$, the GLCM matrix $G m$ can be defined by Riabaric et al., (Ribaric \& Lopar, 2013) as: $G m(i, j, \delta$; $\theta)$, size $G \times G$, where the values of the pixels are quantized into $G$ levels, contains at the position $(i, j)$ a number of occurrences of a pair of pixels that are at the offset $\delta=1,2,3, \ldots$ etc, (the offset $\delta$ is a distance between the pixel-of-interest and its neighbor) in the direction $\theta=0^{\circ}, 45^{\circ}, 90^{\circ}, 135^{\circ}(\theta$ is the angle between the pixel-of-interest and its neighbor or the rotation angle of an offset), where one pixel has a gray-level value $i$ and another pixel has a gray-level value $j$.

In literature, Haralick described fourteen statistical Haralick features to normalize the gray level co-occurrence matrices. In our empirical experiments, we applied only six of these features which are:

- Contrast:

$$
f_{1}=\sum_{n=0}^{G-1} n^{2}\left\{\sum_{i=0}^{G-1} \sum_{j=0}^{G-1} G m(i, j), \quad|i-j|=n\right.
$$

- Variance:

$$
f_{2}=\sum_{i=0}^{G-1} \sum_{j=0}^{G-1}(i-\mu)^{2} G m(i, j)
$$

- Energy:

$$
f_{3}=\sum_{i=0}^{G-1} \sum_{j=0}^{G-1} G m(i, j)^{2}
$$


- Entropy:

$$
f_{4}=-\sum_{i=0}^{G-1} \sum_{j=0}^{G-1} G m(i, j) \times \log (G m(i, j))
$$

- Homogeneity or Inverse difference moment:

$$
f_{5}=\sum_{i=0}^{G-1} \sum_{j=0}^{G-1} \frac{G m(i, j)}{1+(i-j)^{2}}
$$

where $G m$ is GLCM matrix and $G$ is Grey Scale value, $\mu$ is the mean value.

- Correlation:

$$
f_{6}=\sum_{i=0}^{G-1} \sum_{j=0}^{G-1} \frac{(i \times j) \times G m(i, j)-\left(\mu_{x} \times \mu_{y}\right)}{\sigma_{x} \times \sigma_{y}}
$$

where $G m$ is GLCM matrix and $G$ is Grey Scale value, $\mu_{x}, \mu_{y}$ are the mean value and $\sigma_{x,} \sigma_{y}$ are standard deviation along two axes $X$ and $Y$.

In this work, we follow the same parameters proposed by (Mokni, Mezghani, et al., 2017) to construct the feature vector. So, the number of gray levels of palmprint ROI is $G=256$. We have split each ROI into small $8 \times 8$ sub-images by a non-overlapping sliding window. For each ROI, we have obtained 64 sub-images. For each sub-image, the GLCMs matrices are calculated corresponding to four different values of direction with four different offset values mentioned above.

Hence, we have obtained 16 GLCMs (4 (offset value) $\times 4$ (direction number)) for each sub-image. Then, we have calculated the six Haralick features for each GLCM. Therefore, the feature vector is calculated by the multiplication of the number of GLCMs for ROI image and the number of Haralick features, as follows: $F V_{G L C M}=(64 \times 16) \times 6=6144$.

\section{Dimensionality Reduction of Feature Vectors}

Since some of the provided feature vectors have high dimensional features (e.g., $\mathrm{FV}_{\mathrm{HOG}}, \mathrm{FV}_{\mathrm{GF}}$ and $\mathrm{FV}_{\mathrm{GLCM}}$ ), we handle a variety of issues as the increase of the computational complexity and the decrease of the performance accuracy.

In order to overcome these issues, we incorporated a supplementary phase based on applying the dimensionality reduction techniques, such as Principal Component Analysis (PCA) (Jolliffe, 2002) or Generalized Discriminant Analysis (GDA) (Baudat \& Anouar, 2000). These techniques ignore the useless information and thus select the most discriminating of it to reduce the dimension of vector and consequently increase the accuracy and decrease the computational complexity of the proposed identification process based on each methodology. The PCA is an unsupervised technique which aims to map the data space into a linear subspace with lower dimensions and helps find the direction that maximizes the variance in the data. It presents information in an orthogonal linear space.

Nonetheless, the GDA technique is a variant of Linear Discriminant Analysis (LDA) that is supervised technique that encodes pertinent information in a linear space which is not essentially 
orthogonal. It attempts to maximize the separability among the known classes (i.e., it finds the direction that maximizes the difference between two classes). Moreover, GDA technique incorporates the kernel methods which have been famously handled to resolve the pattern recognition issues thanks to their ability to deal with nonlinear data. In our experiments, when applying the GDA technique, we use the RBF as a kernel function with $\sigma=1$.

Ultimately, it is well-known that an approach used the LDA and GDA techniques perform better than that used PCA. However, several proposals ( $c f$., (Sharma \& Paliwal, 2015), (Yu, Yu, \& Xu, 2010), etc.) prove that this performance depends on the size of the training data or the samples for each class. In fact, if the training data set or the sample number is small, the PCA provides better results than the LDA or GDA.

\section{Fusion at Feature Level Based on CCA Technique}

The fusion of different information in the biometric system has gained a great interest in various researchers' teams. This fusion between the multiple descriptors will be improving the recognition accuracy. Indeed, it can occur in different ways for a recognition system, such as: Fusion at the data or feature level, Fusion at the score level and Fusion at the decision level.

Several prior researchers believe that the fusion at feature level way is considered to be more effective than the other ways by virtue of the capability of fusing the multiple sources of information to achieve a single feature set, that contains more efficient richer information than the fusion at the score and the decision levels (Haghighat, Abdel-Mottaleb, \& Alhalabi, 2016). Thus, the fusion at feature level is widely important to produce a substantial identification. There are many manners of the fusion at the feature level, namely: the Serial feature fusion and the Parallel feature fusion. The two fusion manners can ameliorate the accuracy of recognition of persons to some extent.

However, neither of these fusion methods focused on the inherent correlations across multiple feature sets. Therefore, both of these fusion methods cannot reach tremendous feature fusion. To surmount this weakness, the Canonical Correlation Analysis (CCA) feature fusion method is conducted to extract the feature pairs describing the intrinsic relationship between two feature sets (Sun, Zeng, Liu, Heng, \& Xia, 2005). The CCA method firstly implements a function of correlation criterion between two sets of feature vectors and secondly calculates the projection vector set of these two sets depending on the criterion. Finally, the fused canonical correlation features are extracted used either the summation or concatenation of these projection vector sets.

The background and all detail of the CCA Analysis method are described in (Mokni, Mezghani, et al., 2017).

Likewise, the Multi-set Canonical Correlation Analysis (MCCA) method is considered as a generalization of the CCA method and is proposed to be suitable for fusing more than two sets of features, as presented in our cases.

Therefore, the main principle of MCCA method is conducted by combining the first two feature vectors that have highest ranks using CCA method and then fusing the result with the next feature vector that has an utmost rank using CCA method, and so on.

Figure 4 illustrates an example of the process of the MCCA method for four feature vectors with $\operatorname{rank}\left(F V_{1}\right)>\operatorname{rank}\left(F V_{2}\right)>\operatorname{rank}\left(F V_{3}\right)>\operatorname{rank}\left(F V_{4}\right)$.

In this example, the first step of MCCA focuses on fusing $F V_{1}$ and $F V_{2}$, which have the highest ranks using CCA method. Then, $F V_{12}$ is fused with the next highest rank feature vector $F V_{3}$ and finally, $F V_{4}$ is fused with $F V_{123}$ using yet CCA method.

Practically, in this work, we applied the MCCA method incorporating for each fusion between two vectors the CCA method using the concatenation manner to fuse the different features vectors into a single feature vector. 


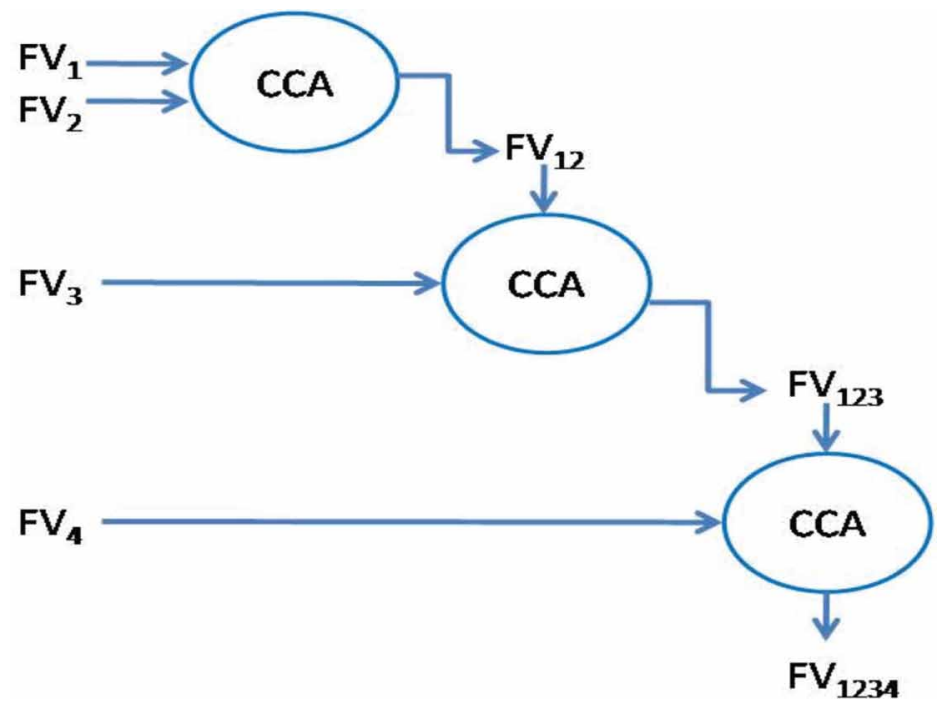

\section{Classification}

For the classification phase, we used the Multi-class version of both the Random Forest (RF) and the Support Vector Machine (SVM) classifiers.

\section{Random Forest Algorithm}

This algorithm was proposed by Leo Breiman in (Breiman, 2001) and defined as a meta-learner comprised of many individual trees. It was designed to quickly operate over large datasets and more importantly to be diverse by using random samples to build each tree in the forest. Diversity is obtained by randomly choosing attributes at each node of the tree and then using the attribute that provides the highest level of learning. Once trained, Random Forest classifies a new person from an input feature vector by putting it down each of the trees in the forest. Each tree gives a classification decision by voting for this class. Then, the forest chooses the classification having the most votes (over all the trees in the forest).

In our experiments, we used Weka Multi-class implementation of Random Forest algorithm by considering 250 trees. A study of effect of the Tree number is mentioned later in the experimental result part.

\section{Support Vector Machine Algorithm}

Support Vector Machine (SVM) is a supervised classifier which is a vigorous statistical technique suggested by Vapnik (Vapnik \& Vapnik, 1998). This classifier has been widely intended to resolve the problems of discrimination, classification or regression in several applications. It is applied to determine an optimal separating hyper-plane or decision surface with the adaptation of a new corresponding technique to map the sample points into a high-dimensional feature space and categorized using a non-linear transformation, even when the data are linearly inseparable.

The optimal hyper-plane is obtained by working out a quadratic programming problem relied on regularization parameters. This transformation was performed by kernel functions like Linear Kernel (LK), Radial Basis Function (RBF) and Polynomial Kernel (PK) which are exploited in this work in order to classify the query sample. For this purpose, we utilized LibSVM toolbox (Chang \& Lin, 2011). These different kernel types defined as: 
- The Linear kernel Function (LK):

$K(x, y)=x \times y$

- $\quad$ The Polynomial Kernel Function (PK):

$K(x, y)=(\gamma \times x y+r)^{d}, \gamma \succ 0$

- $\quad$ The Radial Basis Function (RBF):

$K(x, y)=\exp \left(-\gamma|x-y|^{2}\right), \gamma \succ 0$

where $\gamma, r$ and $d$ are the parameters of the kernel functions that will be determinate empirically.

\section{EXPERIMENTAL EVALUATION AND COMPARISON}

After detailing the proposed approach to analyze the palmprint texture representation in an uncontrolled environment using multiple descriptors, we address, in this section, all the experiments as well as the empirical results coupled with interpretations and discussions on the performances.

The simulation of the proposed system is implemented using MATLAB 2010 on a desktop PC with a $3 \mathrm{GHz}$ Core 2 Duo processor system and $3 \mathrm{~GB}$ memory.

We performed all the experiments of the proposed approach involving three benchmark databases, namely: PolyU-Palmprint (PolyU database, 2003), CASIA-Palmprint (CASIA database, 2005) and IIT-Delhi-Palmprint (IIT Delhi, 2006) databases. Then, we also made a comparison between the accuracy of our proposed approach and the previously well-known methods, for each database.

\section{Palmprint Databases}

\section{PolyU-Palmprint Database}

The Hong Kong Polytechnic University offered a PolyU-Palmprint database that contains 7752 images captured from 386 persons. Twenty samples of each palm were acquired in two separate sessions. Each session contains ten sample images. The average time interval between these sessions is two months. The samples collected during these two separate sessions acquired by two different cameras. This means that there exist variations in terms of lighting between the first and second sessions, which leads to more difficulty in person's identification.

\section{CASIA-Palmprint-Database}

This dataset contains 5502 palmprint images captured from 312 subjects. This dataset is taken from Chinese Academy of Sciences of institution who Automation. For each subject, 8 palmprint images have been collected from both left and right palms. The capture system captures the all palmprint images using a CMOS camera fixed on the top of the device. This latter supplies an evenly distributed illumination on the palmprint images. Since the hand of each person is not touching any platform plate, a presence of distance variation between the hand and the capture device which implies scale variations. In this dataset, the users are not used the guidance pegs to constraint their hands. Therefore, 
these hand images are captured with different directions and postures, which brings on contracting and stretching the texture skin pattern at the region of interest of the palmprint. This dataset has many variations which make the identification of the persons more difficult.

\section{IIT-Delhi-Palmprint Database}

The IIT-New Delhi India campus provides a IIT-Delhi palmprint database that includes 2300 images acquired from 230 persons aged from 12-57 years. For each person, five samples have been collected from both left and right palms. These palmprint samples are captured in unconstrained environment with several challenges and variation in terms of position, scale, direction and texture deformation.

\section{Empirical Results}

We evaluated the accuracy of our proposed approach over three benchmark databases described above. For the PolyU database, we selected ten samples of each person from the first session of the PolyU database for the gallery set and ten samples from the second session were chosen for the probe set. This mixture between the samples collected in separate sessions is important in a real application.

For the CASIA database, we indiscriminately selected 5 samples of each palm as a gallery set and three samples were singled as the probe set.

For the IIT-Delhi database, we arbitrarily singled three samples of each palm as gallery set and the remainder of samples as the probe set.

In our experiments, we conducted the performance of our proposed approach using two different methods of fusion at feature levels, namely: Serial and MCCA methods. For more clearly, the Serial feature fusion is based on combining multiple feature vector sets into a single feature vector using a simple concatenation.

We recall that before fusing the multiple features by MCCA method, we applied the dimensionality reduction based on PCA or GDA method in order to overcome the dimensionality problem. Likewise, when using Serial feature fusion method, we also used the PCA or GDA method to reduce the dimensionality of the final obtained vector.

All the experiments are processed by the classification phase using the SVM and the RF classifiers, which are applied with various kernel functions and number of trees, respectively.

Therefore, we reveal the experiment results of the Serial and MCCA feature fusion methods using PCA and GDA dimensionality reduction techniques, corresponding to several kernel functions of SVM classifier for PolyU and CASIA datasets in Table 1.

It can be noticed that the recognition rates obtained by the SVM classifier with Radial Basis Kernel (RBF) outperforms other kernel functions results (LK and PK) for both datasets.

Since we performed our experiments with both the MCCA and the Serial feature fusion methods, it is obvious that the performance of the MCCA feature fusion method surpasses the Serial feature fusion method for both databases. Noticeably, in this table, the performance of our proposed approach

Table 1. The different results of our proposed deep-analysis approach based on both serial and MCCA method evaluating over PolyU and CASIA databases

\begin{tabular}{|l|l|l|l|l|l|l|}
\hline \multirow{2}{*}{\multicolumn{1}{|c|}{ RRs (\%) }} & \multicolumn{3}{c|}{ PolyU Database } & \multicolumn{3}{c|}{ CASIA Database } \\
\cline { 2 - 7 } & \multicolumn{1}{|c|}{ LK } & \multicolumn{1}{|c|}{ RBF } & \multicolumn{1}{c|}{ PK } & \multicolumn{1}{c|}{ LK } & \multicolumn{1}{c|}{ RBF } & PK \\
\hline Serial + PCA & 96.89 & 97.07 & 59.77 & 96.25 & 97.22 & 55.80 \\
\hline Serial + GDA & 96.91 & 97.12 & 60.05 & 97.44 & 97.99 & 56.09 \\
\hline PCA + MCCA & 97.51 & $\mathbf{9 7 . 7 5}$ & 61.08 & 97.78 & $\mathbf{9 8 . 0 5}$ & 65.20 \\
\hline GDA+MCCA & 97.66 & $\mathbf{9 7 . 8 5}$ & 61.40 & 97.93 & $\mathbf{9 8 . 3 5}$ & 66.04 \\
\hline
\end{tabular}


using GDA-based dimensionality reduction technique outperforms all the results using PCA over PolyU and CASIA databases.

Moreover, in Table 2, we highlight the different recognition rates with Random Forest classification compatible with the various numbers of trees for both the PolyU and CASIA datasets. In this table, we use the MCCA fusion technique at the feature level since it gave the better results in the previous experiments and we highlight the results with PCA and GDA dimensionality reduction techniques.

As shown in this table, the implementation of the Random Forest algorithm with 200 or 250 trees achieves better recognition rates for both databases compared to other numbers of trees. More precisely, it can be inferred that the recognition rates are almost equal starting from 150 trees to 250 trees and then significantly decrease. The performance using GDA technique reaches better results than PCA over both databases.

In addition, in order to further prove the efficiency of the proposed approach, we have assessed it on the IIT-Delhi Palmprint database. Indeed, we present the performance of the experiments under the same conditions as previously, with SVM classifier (RBF) and RF classifier (250 trees), using the MCCA fusion technique at the feature level and applying the PCA and GDA techniques, as reported in Table 3.

Obviously in the Table 3, the performance of our approach using the PCA-based dimensionality reduction technique gives a better accuracy than the GDA technique. This is consistent with several proposals (cf., (Sharma \& Paliwal, 2015), (Qiao, Zhou, \& Huang, 2008), (Martinez \& Kak, 2001), (Yu et al., 2010)), which confirmed that when the training dataset or the number of samples for each class is small, PCA can outperform GDA that is the variant of LDA. Therefore, as the PCA technique tends to give better results if sample number in a given class is slightly small, which is already emphasized in this last aspect when the number of samples for each class in the IIT-Delhi dataset is 5 samples, whereas, in PolyU and CASIA datasets are 20 and 8, respectively.

Furthermore, Table 4 involves not only the achieved recognition rates of the proposed approach but also the execution times relative to preprocessing phase, Feature vectors dimensionality reduction $\&$ Feature Fusion phases (the complexity of the Serial and MCCA feature fusion methods using PCA and GDA dimensionality reduction techniques using their execution-time values) and finally the identification phase. We recall that the dimensionality reduction techniques are used in the proposed approach corresponding to the used dataset protocols (GDA for PolyU and CASIA datasets and PCA for IIT-Delhi dataset).

Table 2. The different recognition rates of our proposed method with random forest algorithm assessing on PolyU and CASIA databases

\begin{tabular}{|c|c|c|c|c|c|c|c|c|c|c|}
\hline \multirow{2}{*}{$\operatorname{RRs}(\%)$} & \multicolumn{5}{|c|}{ PolyU Database } & \multicolumn{5}{|c|}{ CASIA Database } \\
\hline & 100 & 150 & 200 & 250 & 300 & 100 & 150 & 200 & 250 & 300 \\
\hline $\mathrm{PCA}+\mathrm{MCCA}$ & 96.50 & 97.18 & 97.21 & 97.22 & 96.88 & 97.20 & 98.84 & 98.86 & 98.86 & 97.44 \\
\hline GDA+MCCA & 97.12 & 98.28 & 98.30 & 98.30 & 97.25 & 97.50 & 98.92 & 98.97 & 98.98 & 97.62 \\
\hline
\end{tabular}

Table 3. The different recognition rates of our proposed method with random forest algorithm assessing on IITD databases

\begin{tabular}{|c|c|c|c|c|c|c|c|c|c|c|c|c|c|c|c|c|}
\hline \multicolumn{7}{|c|}{ RRs (\%) } & \multicolumn{5}{|c|}{ SVM (RBF) } & \multicolumn{5}{|c|}{ RF (250 Trees) } \\
\hline$P$ & $\mathrm{C}$ & + & M & $\mathrm{C}$ & $\mathrm{C}$ & A & 9 & 7 & . & 3 & 2 & 9 & 7 & - & 9 & 1 \\
\hline G & D A & + & & & $\mathrm{CC}$ & A & 9 & 7 & . & 1 & 5 & 9 & 7 & . & 7 & 7 \\
\hline
\end{tabular}


In this table, we made a comparison between the results obtained using both Serial and MCCA methods and those with dimensionality reduction, as well. Obviously, we showed a gain in term of run-time when using the MCCA fusion method (feature fusion phase) in all databases. It is worth noting that the execution-time values for recognition of one palmprint image in such database averaged over multiple executions. Consequently, it can be inferred that since the MCCA algorithm is only applied the transformations obtained from the training process, it is very fast.

\section{Comparative Results}

To evaluate the performance of our proposed approach with the most popular state of the art approaches, the comparisons over three palmprint databases such as PolyU, CASIA and IIT-Delhi are illustrated in Table 5.

Notice that the presented protocols lie in the sample number during their classification between the gallery and probe data are almost the same over three datasets.

Over IIT-Delhi dataset, it is noted that we achieved a better RR of about $97.91 \%$ than both the presented works in (Kumar \& Shekhar, 2010) $(\mathrm{RR}=95.00 \%)$ and (Mokni, Mezghani, et al., 2017) ( $R R=96.93$ ), which leads an improvement rates of about $2.91 \%$ and $0.98 \%$ to the results of these works.

We compared the result of our proposed approach conducted over CASIA dataset to the works in (Mokni, Drira, \& Kherallah, 2017b) and (Nezhadian \& Rashidi, 2017). Obviously, we obtained a better performance of about $98.98 \%$ than those of the works $(97.00 \%$ and $98.75 \%)$.

Although, all the experiments over PolyU database conducted by a mixture of two sessions, which leads to difficulty for individual's identification, this protocol is very significant in a real application. Noticeably, the result of our proposed method outperforms the totality of prior works results.

In order to make a fair comparison, we assessed our experiments using the same classification of the samples proposed in (Mokni, Drira, et al., 2017b) and (Mokni, Mezghani, et al., 2017) (i.e., ten samples in the gallery set and five in the probe set). Therefore, we reached a recognition rate of about $98.01 \%$, which is still better than the RR in these works. Moreover, we further evaluated our approach following the same evaluation protocols presented in the proposed work by (Wang, Lei, \& Wang, 2012). Hence, it is worth noting that we achieved a better result of about $97.95 \%$ than this work.

\section{Protocols-Setting Description: Gallery Set (G), Probe Set (P)}

These achieved performance evaluating over three benchmark datasets demonstrate the effectiveness and robustness of our proposed method against the different existing challenges and variation produced in an unconstrained environment.

Table 4. The final performance expressed through RR and average execution-time values of our proposed method in PolyU, CASIA and IIT-Delhi databases

\begin{tabular}{|l|l|l|l|l|}
\hline \multicolumn{2}{|l|}{} & \multicolumn{1}{|c|}{$\begin{array}{c}\text { PolyU } \\
\text { Database }\end{array}$} & $\begin{array}{c}\text { CASIA } \\
\text { Database }\end{array}$ & \multicolumn{1}{c|}{$\begin{array}{c}\text { IIT-Delhi } \\
\text { Database }\end{array}$} \\
\hline RRs (\%) & 98.30 & 98.98 & 97.91 \\
\hline \multicolumn{2}{|l|}{ Preprocessing Time (ms) } & 252 & 243 & -- \\
\hline $\begin{array}{l}\text { Feature vectors dimensionality reduction \& } \\
\text { Feature Fusion } \\
\text { Time (ms) }\end{array}$ & Serial \& GDA/PCA & 24 & 23 & 18 \\
\cline { 2 - 5 } & GDA/PCA\& MCCA & 22 & 21 & 18 \\
\hline Identification Time (ms) & 35 & 20 & 10 \\
\hline
\end{tabular}


Table 5. The palmprint recognition rates comparison of our proposed method with the state of-the-art results

\begin{tabular}{|c|c|c|c|}
\hline Proposal & Methods andTechniques & $\begin{array}{l}\text { Palmprint-Datasets } \\
\text { (Protocol-Setting) }\end{array}$ & $\operatorname{RRs}(\%)$ \\
\hline (Kumar \& Shekhar, 2010) & GF & IIT-Delhi (3G/2P) & 95.00 \\
\hline \multirow{2}{*}{ (Mokni, Drira, et al., 2017b) } & \multirow{2}{*}{$\mathrm{FD}, \mathrm{MFD}+\mathrm{LDA}+\mathrm{CCA}+\mathrm{RF}$} & CASIA (5G/3P) & 97.00 \\
\hline & & PolyU (10G/5P) & 96.02 \\
\hline (Nezhadian \& Rashidi, 2017) & $\begin{array}{l}\text { DWT+GLCM + Serial+ FFS + } \\
\text { KNN }\end{array}$ & CASIA (5G/3P) & 98.75 \\
\hline (Wang et al., 2012) & $2 \mathrm{DGW}+\mathrm{PCNN}+\mathrm{SVM}$ & PolyU (5G/10P) & 95.40 \\
\hline \multirow{2}{*}{$\begin{array}{l}\text { (Mokni, Mezghani, et al., } \\
\text { 2017) }\end{array}$} & \multirow{2}{*}{$\begin{array}{l}\text { GF+FD+GLCM +PCA + MCCA } \\
+ \text { SVM (RBF) }\end{array}$} & IIT-Delhi (3G/2P) & 96.93 \\
\hline & & $\begin{array}{l}\text { PolyU (10G/10P) } \\
\text { PolyU (10G/5P) }\end{array}$ & $\begin{array}{l}97.45 \\
97.38\end{array}$ \\
\hline \multirow{3}{*}{ This Proposal } & \multirow{3}{*}{$\begin{array}{l}\mathrm{HOG}+\mathrm{FD}+\mathrm{GLCM}+\mathrm{GDA}+ \\
\mathrm{MCCA}+\mathrm{RF}\end{array}$} & IIT-Delhi (3G/2P) & 97.91 \\
\hline & & CASIA (5G/3P) & 98.98 \\
\hline & & $\begin{array}{l}\text { PolyU (10G/10P) } \\
\text { PolyU (10G/5P) } \\
\text { PolyU (5G/10P) }\end{array}$ & $\begin{array}{l}98.30 \\
98.01 \\
97.95\end{array}$ \\
\hline
\end{tabular}

\section{CONCLUSION}

In this paper, we investigate a deep analysis of palmprint texture representation for a person's identification. This analysis based on the extraction of different and complementary information for texture using multiple descriptors, such as "HOG and GF", "FD" and "GLCM" corresponding respectively to the Frequency, Model and Statistical methodologies-based texture features analysis.

Moreover, we incorporated the dimensionality reduction techniques (PCA and GDA) in order to reduce the feature dimensionality of some of these feature sets and thus improve the performance and decrease the complexity cost of the proposed approach.

Afterward, we fused all obtained texture information using a fusion at feature level based on Multi-set Canonical Correlation Analysis (MCCA) integrating the correlation concept between the texture features, which leads to the improvement of performance of the proposed deep analysis. The experimental results on PolyU-Palmprint", "CASIA-Palmprint" and "IIT-Delhi-Palmprint databases demonstrate the effectiveness of the proposed approach.

As future work, we plan to investigate more evaluation of our proposed approach performance using other biometric evaluation metrics such as the Equal Error Rate (EER), False Accepted Rate (FAR) and False Rejected Rate (FRR) which are the important metrics in the performance estimation of a palmprint verification method.

\section{ACKNOWLEDGMENT}

This Publication was supported by the Deanship of Scientific Research at Prince Sattam bin Abdulaziz University. Raouia Mokni served as the corresponding author throughout the research conducted for this article. 


\section{REFERENCES}

Baudat, G., \& Anouar, F. (2000). Generalized discriminant analysis using a kernel approach. Neural Computation, 12(10), 2385-2404. doi:10.1162/089976600300014980 PMID:11032039

Ben-Khalifa, A., Rzouga, L., \& BenAmara, N. E. (2013). Wavelet, Gabor filters and co-occurrence matrix for palmprint verification. International Journal of Image, Graphics and Signal Processing, 5(8), 1-8. doi:10.5815/ ijigsp.2013.08.01

Breiman, L. (2001). Random forests. Machine Learning, 45(1), 5-32. doi:10.1023/A:1010933404324

CASIA database. (2005). Chinese Academy of Sciences Institute of Automation, Biometrics Ideal Test, Institute of Automation, Chinese Academy of Sciences. Retrieved from http://biometrics.idealtest.org/dbDetailForUser. do?id=5

Chaabouni, A. (2014). Automatic written identification using Fractal and Multi-fractal approaches. Sfax University.

Chang, C.-C., \& Lin, C.-J. (2011). LIBSVM: A library for support vector machines. ACM Transactions on Intelligent Systems and Technology, 2(3), 27. doi:10.1145/1961189.1961199

Chen, J., Zhang, C., \& Rong, G. (2001). Palmprint recognition using crease. In Proceedings of the 2001 International Conference on Image Processing (Vol. 3, pp. 234-237). IEEE.

Dalal, N., \& Triggs, B. (2005). Histograms of oriented gradients for human detection. In Proceedings of the IEEE Computer Society Conference on Computer Vision and Pattern Recognition CVPR 2005. (Vol. 1, pp. 886-893). IEEE. doi:10.1109/CVPR.2005.177

Delhi, I. I. T. (2006). IIT Delhi Touchless Palmprint Database (Version 1.0), Indian Institute of Technology Delhi. Database. Retrieved from http://web.iitd.ac.in/ ajaykr/Database_Palm.htm

Duta, N., Jain, A. K., \& Mardia, K. V. (2002). Matching of palmprints. Pattern Recognition Letters, 23(4), 477-485. doi:10.1016/S0167-8655(01)00179-9

Guo, X., Zhou, W., \& Wang, Y. (2014). Palmprint recognition algorithm with horizontally expanded blanket dimension. Neurocomputing, 127, 152-160. doi:10.1016/j.neucom.2013.08.027

Haghighat, M., Abdel-Mottaleb, M., \& Alhalabi, W. (2016). Fully automatic face normalization and single sample face recognition in unconstrained environments. Expert Systems with Applications, 47, 23-34. doi:10.1016/j. eswa.2015.10.047

Haghighat, M., Zonouz, S., \& Abdel-Mottaleb, M. (2015). CloudID: Trustworthy cloud-based and cross-enterprise biometric identification. Expert Systems with Applications, 42(21), 7905-7916. doi:10.1016/j.eswa.2015.06.025

Hammami, M., Ben-Jemaa, S., \& Ben-Abdallah, H. (2014). Selection of discriminative sub-regions for palmprint recognition. Multimedia Tools and Applications, 68(3), 1023-1050. doi:10.1007/s11042-012-1109-x

Han, C. C., Cheng, H. L., Lin, C. L., \& Fan, K. C. (2003). Personal authentication using palm-print features. Pattern Recognition, 36(2), 371-381. doi:10.1016/S0031-3203(02)00037-7

Haralick, R. M. (1979). Statistical and structural approaches to texture. Proceedings of the IEEE, 67(5), 786-804. doi:10.1109/PROC.1979.11328

Jolliffe, I. T. (2002). Principal Component Analysis. Springer.

Kekre, H. B., Sarode, K., \& Tirodkar, A. A. (2012). A study of the efficacy of using Wavelet Transforms for Palm Print Recognition. In Proceedings of the 2012 International Conference on Computing, Communication and Applications (ICCCA) (pp. 1-6). doi:10.1109/ICCCA.2012.6179174

Kumar, A., \& Shekhar, S. (2010). Palmprint recognition using rank level fusion. In Proceedings of the 2010 17th IEEE International Conference on Image Processing (ICIP) (pp. 3121-3124). doi:10.1109/ICIP.2010.5653214

Latha, Y. L. M., \& Prasad, M. V. N. K. (2015). GLCM Based Texture Features for Palmprint Identification System. In Computational Intelligence in Data Mining (Vol. 1, pp. 155-163). Springer. 
Lee, T.S. (1996). Image representation using 2D Gabor wavelets. IEEE Transactions on Pattern Analysis and Machine Intelligence, 18(10), 959-971. doi:10.1109/34.541406

Lu, G., Zhang, D., \& Wang, K. (2003). Palmprint recognition using eigenpalms features. Pattern Recognition Letters, 24(9), 1463-1467. doi:10.1016/S0167-8655(02)00386-0

Mandelbrot, B. B. (1975). Les objets fractals: forme, hasard et dimension.

Martinez, A. M., \& Kak, A. C. (2001). Pca versus lda. IEEE Transactions on Pattern Analysis and Machine Intelligence, 23(2), 228-233. doi:10.1109/34.908974

Mokni, R., Drira, H., \& Kherallah, M. (2017a). Combining shape analysis and texture pattern for palmprint identification. Multimedia Tools and Applications, 76(22), 23981-24008. doi:10.1007/s11042-016-4088-5

Mokni, R., Drira, H., \& Kherallah, M. (2017b). Fusing Multi-Techniques based on LDA-CCA and their Application in Palmprint Identification System. In Proceedings of the 2017 IEEE/ACS 14th International Conference on Computer Systems and Applications (AICCSA) (pp. 350-357). IEEE. doi:10.1109/AICCSA.2017.167

Mokni, R., Elleuch, M., \& Kherallah, M. (2016). Biometric Palmprint identification via efficient texture features fusion. In Proceedings of the International Joint Conference on Neural Networks (pp. 4857-4864). Academic Press. doi:10.1109/IJCNN.2016.7727838

Mokni, R., \& Kherallah, M. (2016a). Palmprint identification using GLCM texture features extraction and SVM classifier. Journal of Information Assurance \& Security, 11(2).

Mokni, R., \& Kherallah, M. (2016b). Palmprint recognition through the fractal dimension estimation for texture analysis. International Journal of Biometrics, 8(3-4), 254-274. doi:10.1504/IJBM.2016.082599

Mokni, R., Mezghani, A., Drira, H., \& Kherallah, M. (2017). Multiset Canonical Correlation Analysis: Texture Feature Level Fusion of Multiple Descriptors for Intra-modal Palmprint Biometric Recognition. In Proceedings of the Pacific-Rim Symposium on Image and Video Technology (pp. 3-16). Academic Press.

Nezhadian, F. K., \& Rashidi, S. (2017). Palmprint verification based on textural features by using Gabor filters based GLCM and wavelet. In Proceedings of the 2017 2nd Conference on Swarm Intelligence and Evolutionary Computation (CSIEC) (pp. 112-117). Academic Press. doi:10.1109/CSIEC.2017.7940164

Otsu, N. (1979). A threshold selection method from gray-level histograms. IEEE Transactions on Systems, Man, and Cybernetics, 9(1), 62-66. doi:10.1109/TSMC.1979.4310076

PolyU database. (2003). PolyU Palmprint Database, The Biometric Research Centre at The Hong Kong Polytechnic University. Retrieved from http://www4.comp.polyu.edu.hk/ biometrics/

Qiao, Z., Zhou, L., \& Huang, J. Z. (2008). Effective linear discriminant analysis for high dimensional, low sample size data. In Proceeding of the world congress on engineering (Vol. 2, pp. 2-4).

Ribaric, S., \& Lopar, M. (2013). Palmprint Recognition Based on Local Texture Features. In Proceedings of the International Workshop on Intelligent Pattern Recognition and Applications WIPRA2013. Academic Press.

Sharma, A., \& Paliwal, K. K. (2015). Linear discriminant analysis for the small sample size problem: An overview. International Journal of Machine Learning and Cybernetics, 6(3), 443-454. doi:10.1007/s13042-013-0226-9

Sun, Q. S., Zeng, S. G., Liu, Y., Heng, P. A., \& Xia, D. S. (2005). A new method of feature fusion and its application in image recognition. Pattern Recognition, 38(12), 2437-2448. doi:10.1016/j.patcog.2004.12.013

Vapnik, V. N., \& Vapnik, V. (1998). Statistical learning theory (Vol. 1). Wiley New York.

Wang, X., Lei, L., \& Wang, M. (2012). Palmprint verification based on 2D-Gabor wavelet and pulse-coupled neural network. Knowledge-Based Systems, 27, 451-455. doi:10.1016/j.knosys.2011.10.008

Wu, X., Zhang, D., \& Wang, K. (2003). Fisherpalms based palmprint recognition. Pattern Recognition Letters, 24(15), 2829-2838. doi:10.1016/S0167-8655(03)00141-7

Yu, P., Yu, P., \& Xu, D. (2010). Comparison of PCA, LDA and GDA for palmprint verification. In Proceedings of the 2010 International Conference on Information Networking and Automation (ICINA) (Vol. 1, pp. V1-148). 
Zouari, R., Mokni, R., \& Kherallah, M. (2014). Identification and verification system of offline handwritten signature using fractal approach. In Proceedings of the 2014 First International Image Processing, Applications and Systems Conference (IPAS) (pp. 1-4). IEEE. doi:10.1109/IPAS.2014.7043305

Raouia Mokni was born in Sfax (Tunisia) in 1986. She graduated with a master's thesis in computer science from the University of Sfax-Tunisia in 2011. Currently, she is preparing her Ph.D at the University of Sfax in Tunisia (FSEGS) and she is a Teaching-Assistant at the ISGIS in University of Sfax. She is an IEEE member. Her research interests include Biometrics, Pattern recognition, and image processing.

Hassen Drira received the engineering and M.Sc. degrees in Computer Science from National School of Computer Science (ENSI), Manouba, Tunisia, in 2006 and respectively 2007, and the Ph.D degree in Computer Science in 2011, from University Lille 1, France. After spending the year 2011-2012 at the MIIRE research group within the CRIStAL laboratory of Lille (UMR CNRS 9189) France, he joined Institut Mines-Télécom/Télécom Lille as an Associate Professor in 2012. His research interests are mainly focused on pattern recognition, statistical analysis and recognition of $3 D$ faces, biometric, and expression recognition.

Monji Kherallah He was born in Sfax (Tunisia) in 1963. He graduated in Electrical Engineering 1989, obtained a Ph.D. in Electrical Engineering in 2008. He is now a professor in Electrical \& Computer Engineering at the University of Sfax. His research interest includes applications of intelligent methods to pattern recognition and industrial processes. He focuses his research on handwritten documents analysis and recognition, handwritten Arabic recognition, biometrics, pattern recognition and image processing. He is a member of the editorial board of "Pattern Recognition Letters." He was a member of the organization committee of several International Conferences as ACIDCA-ICMI'2005, chair of ACIT'2017, etc. 